\title{
UPDATE AND GUIDANCE ON MANAGEMENT OF MYOPIA. EUROPEAN SOCIETY OF OPHTHALMOLOGY IN COOPERATION WITH INTERNATIONAL MYOPIA INSTITUTE
} \author{
H. Radhakrishnan ${ }^{25}$, S. Resnikoff ${ }^{26,27}$ \\ ${ }^{1}$ Department of Ophthalmology, Semmelweis University, Budapest, Hungary \\ ${ }^{2}$ Faculty of Health Sciences, Semmelweis University, Budapest, Hungary \\ ${ }^{3}$ King's College Hospital, London, UK \\ ${ }^{4}$ Department of Ophthalmology, University of Ghent, Ghent, Belgium \\ ${ }^{5}$ Department of Ophthalmology, Heidelberg University, Mannheim, Germany \\ ${ }^{6}$ Rotterdam Eye Hospital, Rotterdam, The Netherlands \\ ${ }^{7}$ Lithuanian University of Health Sciences, Kaunas, Lithuania \\ ${ }^{8}$ Department of Ophthalmology, University of Warmia and Mazury, Olsztyn, Poland
}

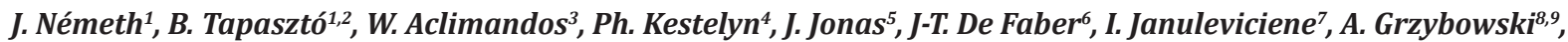
Z. Zsolt Nagy ${ }^{1}$, O. Pärssinen ${ }^{10}$, J. Guggenheim ${ }^{11}$, P. Allen ${ }^{12}$, R. Baraas ${ }^{13}$, K. Saunders ${ }^{14}$, D. Flitcroft1 ${ }^{5,16}$, L. Gray $^{17}$, J. Polling ${ }^{18,19}$, A. Haarman ${ }^{18}$, J. Tideman ${ }^{18}$, J. Wolffsohn ${ }^{20}$, S. Wahl21,22, J. Mulder ${ }^{19}$, I. Smirnova ${ }^{23}$, M Formenti ${ }^{24}$,

\begin{abstract}
The prevalence of myopia is increasing extensively worldwide. The number of people with myopia in 2020 is predicted to be 2.6 billion globally, which is expected to rise up to 4.9 billion by 2050 , unless preventive actions and interventions are taken. The number of individuals with high myopia is also increasing substantially and pathological myopia is predicted to become the most common cause of irreversible vision impairment and blindness worldwide and also in Europe. These prevalence estimates indicate the importance of reducing the burden of myopia by means of myopia control interventions to prevent myopia onset and to slow down myopia progression. Due to the urgency of the situation, the European Society of Ophthalmology decided to publish this update of the current information and guidance on management of myopia. The pathogenesis and genetics of myopia are also summarized and epidemiology, risk factors, preventive and treatment options are discussed in details.
\end{abstract}

Key words: myopia, pathologic myopia, preventive medicine, blindness, time spent outdoors, myopia reduction interventions, atropine, orthokeratology

\section{АКТУАЛНИ НАСОКИ В КОНТРОЛА И ПРЕВЕНЦИЯТА НА МИОПИЯТА ОТ ЕВРОПЕЙСКОТО ОФТАЛМОЛОГИЧНО ОБЩЕСТВО И МЕЖДУНАРОДНИЯ ИНСТИТУТ ПО МИОПИЯ}

\footnotetext{
Резюме. Честотата на миопията се увеличава изключително много в световен мащаб. Броят на хората с късогледство до 2020 г. е от порядъка на 2.6 млрд. души, а до 2050 г. се смята, че броят им ще нарасне до 4.9 млрд. души, освен ако не се предприемат необходимите мерки за превенция. Броят на пациентите с високостепенна миопия също значително нараства и се предполага, че патологичната миопия в бъдеще ще бъде най-честата причина за невъзвратима загуба на зрение и слепота както в Европа, така и по света. Тази статистика показва важността да се намали броят на патологичната миопия чрез различни профилактични мерки, както и да се намали скоростта на прогресия на късогледството. Поради важността на проблема Европейското офталмологично общество реши да публикува актуалните препоръки за превенция и контрол на миопията. Патогенезата, епидемиологията и рисковите фактори, както и терапевтичните възможности са дискутирани в детайли.
}

Ключови думи: миопия, патологична миопия, превантивна медицина, слепота, занимания на открито, интервенции за редукция на миопията, атропин, ортокератология 


\section{INTRODUCTION}

Myopia is recognized as a significant global public health issue, expected to affect an increasing number of people in the next decades. ${ }^{1} \mathrm{~Pa}$ thology such as myopic maculopathy and optic neuropathy in highly myopic eyes can cause significant irreversible visual impairment and blindness. ${ }^{1-3}$ Besides, myopia increases the risk of other pathological ocular changes such as cataract, glaucoma, and retinal detachment, all of which can cause irreversible vision loss. ${ }^{4,5}$ Significant disease associations exist even at low and moderate levels of myopia. Current evidence suggests that there is no safe threshold level of myopia for any of the known ocular diseases linked to myopia. 6,7

The importance of the increase in the prevalence of myopia and its sequelae has been well documented particularly in East Asian countries that have experienced the most pronounced increase in myopia prevalence. Consequently, the experience in preventing the development and progression of myopia in children and adolescents is more advanced in East Asia. The prevalence of myopia also increased in Europe in recent decades and reached the level of $45-50 \%$ in the 25-29 years old age group. ${ }^{8}$ Thus, Europe is becoming aware of the importance of myopia both as a public health issue and as a significant socioeconomic burden.

The International Myopia Institute (IMI) with its group of 85 multidisciplinary experts in the field has recently published a series of white papers on the pathogenesis of myopia including the results of experimental studies, ${ }^{9}$ genetics, ${ }^{10}$ and the results of clinical studies including the outcome of randomized controlled trials. ${ }^{11}$ Based on the IMI White Papers, ${ }^{11}$ the main aim of this article is to increase awareness and to provide recommendations for European ophthalmologists to prevent the development and progression of myopia in children and adolescents.

\section{DEFINITION AND CLASSIFICATION}

To be consistent with international standards, the definitions and classification of myopia as used in this article follow those described by the IMI in the IMI White Papers.

\section{EPIDEMIOLOGY}

According to Holden et al. ${ }^{1}$ quoted in the World Report on Vision published by the World Health Organization (WHO) in October 2019, the estimated number of people globally with myopia in 2020 was predicted to be 2620 million, with a further expected increase to 3361 million by 2030. The number of individuals with high myopia was also expected to increase substantially from 399 million in 2020 to 516 million by $2030{ }^{1,46}$ Both these estimates assume no impact of interventions intended to slow down myopia progression.

This means that pathological myopia is predicted to become the most common cause of irreversible vision impairment and blindness worldwide, and the prevalence estimates indicate the importance of reducing the global burden of myopia by means of myopia reduction interventions

The predicted prevalence of myopia by 2050 is $65 \%$ of the population in Asia, $56 \%$ in Western Europe, 54\% in Central Europe, and 50\% in Eastern Europe ${ }^{1}$.

Many studies have reported substantial variations in the prevalence of myopia between different ethnic groups and different age groups. ${ }^{47}$

\section{PREVALENCE OF MYOPIA IN DIFFERENT ETHNIC GROUPS}

The myopia burden is highest in East Asia and the high-income countries of the Asia-Pacific region (51.6\% and 53.4\% prevalence in 2020, respectively) but the prevalence is also high in Europe (Western Europe: 36.7\%, Central Europe: 34.6\%, and Eastern Europe: 32.2\%). ${ }^{1,48}$ An earlier meta-analysis from fifteen population-based adult cohorts and cross-sectional studies across Europe determined an age-standardised prevalence of $30.6 \%$ for myopia. ${ }^{49}$ The peak prevalence of myopia was identified in the 25-29 years age group (47.2\%) although the prevalence of high myopia was relatively low in Europe, with an agestandardised estimate of $2.7 \%{ }^{49}$

A recent review by Grzybowski et al. showed that the prevalence of myopia in school-aged children was $73 \%$ in East Asia and $42 \%$ in North America. A low prevalence (under 10\%) was described in African and South American children. ${ }^{8}$ 
Table 3. Prevalence of myopia in children in Europe.

\begin{tabular}{|l|c|c|c|c|c|c|}
\hline Authors & Publication time & Age (years) & Location & $\begin{array}{c}\text { Number of } \\
\text { participants }\end{array}$ & $\begin{array}{c}\text { Definition } \\
\text { (diopter) (D) }\end{array}$ & $\begin{array}{c}\text { Myopia } \\
\text { prevalence (\%) }\end{array}$ \\
\hline Matamoros $^{51}$ & 2015 & $0-9$ & France & 1781 & $\leq-0.5$ & 19.6 \\
\hline Tideman $^{52}$ & 2017 & 6 & Netherlands & 5711 & $\leq-0.5$ & 2.4 \\
\hline Enthoven et al. $^{53}$ & 2020 & 9 & Netherlands & 5074 & $\leq-0.5$ & 11.5 \\
\hline Rudnicka et al. $^{54}$ & 2010 & $10-11$ & United Kingdom & 233 & $\leq-0.5$ & 3.4 \\
\hline O'Donoghue et al. $^{55}$ & 2015 & $12-13$ & Northern Ireland & 661 & $\leq-0.5$ & 17.7 \\
\hline Tideman et al. $^{56}$ & 2020 & 13 & Netherlands & 3600 & $\leq-0.5$ & 22.2 \\
\hline Matamoros $^{51}$ & 2015 & $10-19$ & France & 8289 & $\leq-0.5$ & 42.7 \\
\hline Lundberg $^{57}$ & 2017 & Mean: 15.4 & Denmark & 307 & $\leq-0.5$ & cycloplegia: 17.9 \\
\hline Hagen et al. $^{58}$ & 2018 & $16-19$ & Norway & 393 & $\leq-0.5$ & 13 \\
\hline
\end{tabular}

Table 4. Prevalence of myopia in teenage Asian children.

\begin{tabular}{|c|c|c|c|c|c|c|}
\hline Authors & $\begin{array}{c}\text { Publication } \\
\text { time }\end{array}$ & Age (years) & Location & $\begin{array}{c}\text { Number of } \\
\text { participants }\end{array}$ & $\begin{array}{c}\text { Definition } \\
\text { (diopter) (D) }\end{array}$ & $\begin{array}{c}\text { Myopia } \\
\text { prevalence } \\
(\%)\end{array}$ \\
\hline Lam et al. ${ }^{59}$ & 2004 & $13-15$ & Hong Kong & 289 & $\leq-0.5$ & 87.2 \\
\hline Matsumara et al. ${ }^{60}$ & 1999 & 17 & Japan & 346 & $\leq-0.5$ & 66.0 \\
\hline Wu et al. ${ }^{61}$ & 2013 & 17 & China & 6026 & $\leq-0.5$ & 84.6 \\
\hline Lin et al. ${ }^{62}$ & 2004 & $16-18$ & Taiwan & 2474 & $<-0.25$ & 84.0 \\
\hline Jung $^{63}$ & 2012 & 19 & South Korea & 23,616 & $<-0.5$ & 96.5 \\
\hline Lee et al. ${ }^{64}$ & 2013 & 19 & South Korea & 2805 & $\leq-0.5$ & 83.3 \\
\hline Koh et al. ${ }^{65}$ & 2014 & Mean: 19.8 & Singapore & 28,908 & $<-0.5$ & 81.6 \\
\hline
\end{tabular}

In groups of White ethnicity there was no clear evidence of differences in myopia prevalence between studies of recent decades from Europe, the USA and Oceania. ${ }^{50}$ (Tables 3 and 4).

\section{Prevalence OF Myopia ACROSS Age GROUPS}

An early age of onset of myopia appears to be the strongest predictor of high myopia in both Asians and White children..$^{71-74}$

It was recently shown that parental myopia was associated with a greater risk of early-onset myopia in Asian, Hispanic, non-Hispanic white, and African American children. ${ }^{75}$

In children younger than 6 years of age the prevalence of myopia is low. This is the case even in Asia where the prevalence of myopia is considered to be critically high in young adults (Table 5).

The prevalence of myopia increases markedly from approximately 6 years of age. When comparing the indicators for the last 45 years (from 1971 to 2016), the frequency of myopia in Novosibirsk school-children aged 7-10 years increased 5.1 times (from 4.5\%to 23.0\%), 11-14 years increased 3.8 times (from $10.5 \%$ to $40.0 \%$ ), and 15-18 years increased 2.1 times (from $21.5 \%$ to $45.0 \%)$. It follows that the highest rate of onset of myopia is currently observed in children aged 7 to 10 years. $^{79,80}$ In the Correction of Myopia Evaluation Trial (COMET) study 426 ethnically diverse (African, Asian, Hispanic,Mixed, and White) myopic children were followed-up annually for 
Table 5. Prevalence of myopia in younger ages (<9 years)

\begin{tabular}{|c|c|c|c|c|c|c|}
\hline Authors & $\begin{array}{c}\text { Publication } \\
\text { time }\end{array}$ & Age (years) & Location & $\begin{array}{c}\text { Number of } \\
\text { participants }\end{array}$ & $\begin{array}{c}\text { Definition } \\
\text { (diopter) (D) }\end{array}$ & $\begin{array}{c}\text { Myopia } \\
\text { prevalence (\%) }\end{array}$ \\
\hline Ma et al. ${ }^{76}$ & 2016 & 3 & Shanghai, China & 8267 & $\leq-0.5$ & 1.8 \\
\hline Guo et al..$^{77}$ & 2017 & 3 & China & 1127 & $\leq-0.5$ & 0.0 \\
\hline Wu et al. ${ }^{61}$ & 2013 & 4 & China & 6026 & $\leq-0.5$ & 1.2 \\
\hline Li et al. ${ }^{78}$ & 2014 & $5-9$ & Northern China & 1675 & $\leq-0.5$ & 0.9 \\
\hline
\end{tabular}

at least 6 and up to 11 years to assess, amongst other things, when myopia seemed to stabilize. Nearly half $(48 \%)$ of the children had stable myopia by age 15 years, $77 \%$ by 18 years and $90 \%$ by the age 21 years. There is evidence for myopia to have stabilized in most myopic individuals by the age 24 years, except in high myopes. ${ }^{83-85}$ In a 23 year follow-up study of Finnish myopic children from mean age of 11 years at baseline, the adulthood myopic progression from the mean age of 24 years 8 years onwards was $\geq 1.00 \mathrm{D}$ in $17.9 \%$ of cases and mean annual change was $-0.05 \pm$ $0.09 \mathrm{D}^{86}$

Other studies show a decline in progression rate with increasing age in young myopes of both European and Asian ethnicity. ${ }^{69,87,88}$ In White European children, average yearly progression rates for myopia and axial length were $-0.41 \mathrm{D}$ and $-0.30 \mathrm{~mm}$ respectively between 6 and 16 years of age and $-0.16 \mathrm{D}$ and $0.15 \mathrm{~mm}$ respectively between 12 and 22 years. ${ }^{88}$

In 2000, Holden et al. showed that the greatest proportion of myopic people was between 10 and 39 years of age. ${ }^{1} \mathrm{~A}$ European study also reported that myopia was most common in younger participants (47.2\%), with those aged $25-29$ years having a prevalence almost double $(27.5 \%)$ that of those of middle and older age (55-59 years). ${ }^{89}$

However, given the predicted increases in prevalence discussed above, the distribution of myopia in the population is expected to widen by 2050 , with a significant proportion of the population exhibiting myopia from 10 years of age all the way through to 79 years of age; with the bulk of late onset (16 years or older) myopia, reflecting the significant lifestyle changes, mostly intensive near work over the past 10 to 25 years. ${ }^{1,73,90}$ This may well be exacerbated by changes in working patterns following the Covid-19 pandemic (increased time indoors, increased time on electronic devices, etc.).
Based on a meta-analysis from 2015 , there is a clear trend of higher myopia prevalence in the last 20 to 30 years across Western and Northern Europe. ${ }^{89,91}$ In contrast, while the prevalence of myopia is reported to have been rising around the world, a similar trend in Southeast Norway appears to be absent. ${ }^{58}$ Neither in Denmark, where nearly 140 years of myopia research did not find a convincing change in prevalence of myopia. ${ }^{92}$ Asian ancestry does not inevitably lead to myopia, since the prevalence of myopia in these areas was much lower two or three generations ago. ${ }^{88,93}$ This suggests that environmental and social factors must be involved in the promotion of myopia in modern populations.

At the current time, the incidence of myopia is increasing in younger age groups, which means that prevalence rates in older adults are generally lower. ${ }^{47,51,91,94}$ However, a bimodal pattern was observed in the prevalence of myopia among subjects of African-American ethnicity and in the population of Singapore. In both groups aged 40+ years, the prevalence was also highest among individuals in their forties and seventies. ${ }^{95,96} \mathrm{~A}$ similar bimodal pattern was found in France, but the peak of myopia prevalence was in people in their twenties and above eighties. ${ }^{51}$ The bimodal distribution is probably due to the increase of the axial myopia among younger people, and secondary refractive myopization due to nuclear cataract in elderly people. ${ }^{47}$

\section{INTERVENTIONS FOR CONTROLLING MYOPIA}

The main measures that can be taken for the prevention of the development of myopia and for the reduction of the progression of myopia include: (1) Public health (lifestyle) interventions - optimization of environmental influences, (2) Pharmacological approach with the topical appli- 
cation of atropine eye drops, (3) Optical devices including multifocal spectacles and multifocal contact lenses that can have aspheric or discrete dual-focus designs, and orthokeratology.

There is high quality evidence that all methods slow the development or progression of myopia although the efficacy is different for the various interventions.

\section{OPTIMIZATION OF ENVIRONMENTAL INFLUENCES}

Outdoor activities. Many studies (including randomized clinical trials) highlight the protective role of increased outdoor/sport time on myopia prevention. ${ }^{115,155,157,172-177}$ In a meta-analysis, every additional hour of outdoor time per week lead to a reduction in the risk of myopia by $2 \% .{ }^{178}$ The chance of becoming myopic is reduced by around one third if time spent outdoors is increased from 0 to $5 \mathrm{~h}$ per week to 14 or more hours per week. ${ }^{172,179}$

The mechanism of increased outdoor time as an intervention for myopia control is not completely clear. Spending time outdoors itself, instead of physical activities outdoors, has been suggested to be the protective factor. ${ }^{150,180}$ Patterns of defocus on the retina by three-dimensional structures of the environment have also been proposed as a possible mechanism of protection during outdoor activities. ${ }^{6}$

The protective effect of outdoor activity on myopia development in children seems to be partly mediated by the light-stimulated release of dopamine from the retina, since increased dopamine release appears to inhibit increased axial elongation. ${ }^{179,181}$ The absence of ultraviolet (UV) light may provoke axial myopia. ${ }^{182}$ According to Flitcoft et al., compared to the spatial properties of the natural world, man-made (urban) environments and indoor environments have spatial features similar to those than created by diffusing filters that induce form deprivation myopia in animal models. ${ }^{163}$ The spatial frequency composition of the constructed environment, both indoors and outdoors, is therefore different from the natural world. Enhancing spatial frequency content of the visual scene may help to limit myopia.
Evidence linking time outdoors to the prevention of myopia is stronger than that linking it to slowing the progression of existing myopia. ${ }^{179}$

Wu et al. have shown that participation in outdoor activities during school recess (10-20 min in both the morning and afternoon) has a significant effect on myopic shift in non-myopic children but not on the myopic progression of children with myopia. ${ }^{175}$ Confirming the above relationship, another study did not detect an effect of near work or time outdoors on the progression of myopia in those with established myopia. ${ }^{174}$ However, other studies have shown faster myopia progression during the darker winter than the brighter summer months. ${ }^{183,184}$

Vitamin D. A number of studies have reported lower levels of serum vitamin D in myopes compared with non-myopes. ${ }^{185-189}$

Lower 25-hydroxyvitamin D concentration in serum was associated with longer AL and a higher risk of myopia in young children, and the effect was independent of outdoor exposure time. Associations were not different between European and non-European children. ${ }^{185}$ In another study, total vitamin D and D3 were biomarkers for time spent outdoors, however there was no evidence they were independently associated with future myopia. ${ }^{190}$

In a study by the CREAM consortium, a Mendelian randomization analysis did not support a direct involvement of vitamin $\mathrm{D}$ with myopic refractive error, as individuals genetically predisposed to lower 25(OH)D levels were not more myopic. ${ }^{152}$

Indoor lighting. In a Chinese study, increasing the light levels from approximately 100 to 500 lux in school class-rooms had a significant effect on myopia onset, refraction and axial elongation. ${ }^{149}$ Another more recent multivariate logistic analysis reported that time spent with a light intensity of $>3000$ lux was a protective factor for myopia in China. ${ }^{158}$

Studies are investigating if achieving light levels indoors similar to the outdoor environment can reduce the incidence and progression of myopia. ${ }^{163,191}$ Torii et al. examined short wavelength violet (360-400 $\mathrm{nm}$ wave-length) light which is absent in indoor environments and may play a role in the inhibition of myopia progression. ${ }^{151}$ 
They showed that over a 1-year period, children who wore violet light transmitting contact lenses had significantly less axial length elongation compared to those wearing violet light blocking eyeglasses. ${ }^{151}$

During the last few years, light-emitting diode (LED) lights have been designed as a new generation of task lights instead of traditional light sources. A cross-sectional-study, based in China, determined the association of the types of lamp for homework (including incandescent lamp, fluorescent lamp, and LED lamp) with the prevalence of myopia in young teenagers. Using LED lamps was associated with more myopic refractive error and longer axial length. ${ }^{192}$

Moreover, the French Agency for Food, Environmental and Occupational Health and Safety (ANSES) recommended avoiding the use of LED light sources emitting cold-white light with a strong blue component in places frequently used by children, to prevent possible photochemical damages and photoreceptors loss. ANSES recommends limitation of the sale of LEDs for domestic use. ${ }^{193}$

Conclusion environmental influences: Near work indoor and outdoor activity play important roles in the development of myopia and in the prevention of myopia, respectively. There is strong evidence that less near work and more outdoor activity provide protection against myopia development in the human eye.

Time outdoors itself, rather than physical activity outdoors, has been suggested to be the protective factor. ${ }^{150,180}$ The link between time outdoors in the prevention of myopia is stronger than the link between time outdoors and slowing of the progression of existing myopia. ${ }^{179}$

\section{OPTICAL}

Spectacles. Wearing spectacles is non-invasive and generally well-tolerated.

Undercorrection. Undercorrection of myopia with spectacles has been common practice for many years. The theory is to reduce myopia progression by reducing the accommodative demand during near work. Current evidence suggests this is not beneficial and can be harmful.

An early non-randomized trial from 1965 found that undercorrection slowed the progression of myopia. ${ }^{194}$
In another study from 2017, over a period of 2 years, 12-year-old Chinese children with no correction had slower myopia progression (diff: $0.29 \mathrm{D}$ ) and less axial elongation $(0.08 \mathrm{~mm})$ than children with full correction suggesting myopic defocus might act as an inhibitor of eye growth in humans. ${ }^{195}$

However, other studies examining undercorrection found just the opposite, namely either an increase in myopia progression or significantly more baseline myopia and longer axial length in children with undercorrection than in children with full correction. ${ }^{196-198}$

A 1-year study of myopic Chinese children, wearing spectacles which either under- or fully corrected their myopia did not show any differences in myopia progression or axial elongation. ${ }^{198}$

Undercorrection strategies do not provide optimal distance visual acuity and may also lead to behavioural changes, such as a reduction in outdoor activities in some children which, as noted above, may promote myopia progression. ${ }^{199}$

As also summarized in the recent Cochrane and other systematic reviews, an over-correction or under-correction of the myopic refractive error had no strong evidence of benefits and instead possible risks for myopia progression200-202 and should be avoided.

Peripheral defocus-correcting spectacle lenses. Studies have assessed different types of novel spectacle lens designs aimed at modulating the relative peripheral defocus in Asian children, with no differences in the rate of progression of myopia or axial elongation when compared with single vision (SV) control groups. ${ }^{203}$ Aspherization of the distance zone added to progressive additional lenses (PALs) did not enhance their therapeutic efficacy in slowing myopia progression. ${ }^{204}$

Moreover, novel spectacle lens design to reduce peripheral hyperopic defocus was reported to demonstrate a reduction in myopia progression in the younger subgroup of children aged 6 to 12 years with a parental history of myopia, in a 1-year trial. ${ }^{205}$

However, this beneficial effect was only observed in an exploratory subgroup analysis that had insufficient statistical power to produce definitive results.

More recently, a specially designed 'competing defocus' spectacle lens, called Defocus In- 
corporated Multiple Segments (DIMS) spectacle lens has been used for myopia control in a 2-year randomized trial by Lam et al. ${ }^{206}$ This lens design has a central optical zone for correcting refractive error and multiple segments of constant myopic defocus (+3.50 D) surrounding the central zone. This enables the lens to provide clear vision and myopic defocus simultaneously for distance, intermediate or near. The results from the clinical trial showed that children of East Asian ethnicity wearing DIMS lenses had 52\% less myopia progression (average $-0.41 \pm 0.06 \mathrm{D}$ in the DIMS group vs. average $-0.85 \pm 0.08 \mathrm{D}$ in the single vision group) and $62 \%$ less axial elongation (mean difference $0.34 \pm 0.04 \mathrm{~mm}$ ) compared with single vision spectacle lenses and about $21.5 \%$ of the DIMS lens wearers had no myopia progression during the 2-year long study period while among the controls this was the case in only $6 \%{ }^{206}$

Conclusion spectacle lenses: Undercorrection of myopia is not recommended as it increased myopia progression slightly (low-certainty evidence, Cochrane-2020) ${ }^{201}$ and did not slow myopia progression as previously thought. Bifocal spectacles or progressive addition lenses versus single vision lenses (SVLs) yielded a small effect in slowing myopia progression (moderatecertainty evidence). ${ }^{201}$ Studies evaluating different peripheral defocus-correcting lenses versus SVLs reported inconsistent results for refractive error and axial length outcomes (low-certainty evidence) ${ }^{201}$ although results for DIMS spectacles are promising. ${ }^{201}$

\section{Contact lenses}

Soft contact lens (SCL). There is no substantial evidence in the literature that conventional soft contact lens wear leads to either slower or faster myopia progression than spectacle wear. ${ }^{199,}$ 216${ }^{218}$ Rigid gas permeable (RGP). In some studies, rigid gas permeable lenses were reported to slow myopia progression in children, ${ }^{219-221}$ but more recent, well-designed studies showed that the use of these lenses did not impact axial elongation and that the apparent control of myopia progression observed with RGPs was most likely induced by temporary corneal flattening. ${ }^{222,223}$

\section{GUIDELINES FOR CLINICAL MANAGEMENT AND CONTROL OF MYOPIA IN CHILDREN}

\section{Selection of myopia control methods}

Based on the child's individual and parental factors, it should be possible to offer a strategy against myopia progression. Decision to treat should be based on age of onset and axial length or refraction at a given age. ${ }^{285}$ If there is a suspicion of any underlying ocular disease, additional examinations are recommended (corneal topography, electrophysiology, retinal imaging, or genetic testing). ${ }^{337}$

\section{Lifestyle advices}

Indoor and near work activity. Excessive near work may influence the development and progression of myopia. ${ }^{11,100,340}$ Close reading distance (20-25 cm) and continuous reading (> $45 \mathrm{~min})$, head tilt, closer nib-to-fingertip distance (which means greater head tilt) have been associated with greater odds of myopia progression. ${ }^{158,340-342}$

In a 23-year follow-up study of Pärssinen, myopic progression was highest among those whose reading posture in childhood was sitting and lowest among those who reported reading in suppine position. ${ }^{343}$ Short reading distance in childhood predicted higher adulthood myopia among females. Time spent on reading and close work in childhood was related to myopic progression during the first 3 years but did not predict adulthood myopia. ${ }^{86}$

Children should not be prevented from participating in near work activity, but attention should be given to the following measures: regular breaks, appropriate reading distances without head tilt, and near-to-distance fixation changes while reading with sufficient outdoor activities. $^{339}$ The Erasmus Myopia Research Group in the Netherlands recommends complete absence of close-up screen use for children up to 2 years old; maximum 1 h day, for children up to 5 years, and a maximum of $2 \mathrm{~h}$ day for children aged 5-12 years. ${ }^{179,337,344}$

Time spent outdoors and lighting. Spending time out-doors without requiring physical activity or direct sunlight exposure appears to have a 
protective effect against myopia onset but not for myopic progression. ${ }^{86,153,339}$

Every additional 1-h of outdoor time per week is associated with a reduction in the risk of myopia by $2 \% .{ }^{178}$ The chance of becoming myopic is reduced by around one third if time spent outdoors is increased from 0 to $5 \mathrm{~h}$ per week to 14 or more per week. ${ }^{172,179}$

A minimum of 8 to $15 \mathrm{~h}$ of outdoor activity per week is recommended for school aged children to achieve clinically meaningful protection from myopiagenic stimuli. ${ }^{157,172,174,176,178,179,345}$ Individuals who are at risk of developing myopia should try to maximise natural lighing and to increase time spent outdoors. ${ }^{149,150,158,339}$ To maximise indoor lighting ${ }^{149,150,339}$ use incandescent light bulbs rather than fluorescent or LED lighting. ${ }^{192,334}$

Nutritional advice. Nowadays a lot of health issues may be connected to nutritional habits. Therefore, parents may enquire whether a change in dietary habits could decrease the probability of eye diseases.

In Chinese schoolchildren higher saturated fat and cholesterol intake were associated with longer axial length. ${ }^{346}$ As noted above, treatment with caffeine metabolite seven-methylxanthine has small effect on eye growth in children. ${ }^{302}$

Although caffeine-like stimulants may be part of nutritional advice for myopes in the future, there is no current high-level evidence to support nutritional treatments for myopia control. ${ }^{339}$

Refractive corrections. Spectacle lenses are non-invasive, simple, and affordable technique for optical correction of refractive errors, such as myopia.

Children should be encouraged to wear their myopic correction full time, as undercorrection of myopia has been shown in some studies to increase myopia progression. ${ }^{196,197,198}$

Decreasing full distance myopic refractive error correction during near work will reduce accommodative demand and accommodative lag. ${ }^{339}$ However, in a 3-year randomized controlled clinical trial mildly myopic school children aged 9-11 years showed significantly less myopia progression when they wore full correction continuously than wearing spectacles only for distant vision. Neither the use of bifocals nor avoiding the use of spectacles in reading slows myopia progression. ${ }^{211}$ Spectacle with peripheral defocus designs such as the DIMS lenses ${ }^{206}$ should be considered over SV lenses in progressing myopes.

Contact lenses play an important role in myopia control. This includes ortho-K and regulatory approved soft contact lenses for myopia control, and studies are ongoing comparing the effect on myopia control of various recently developed contact lens types. Based on a meta-analysis, ortho-k and soft lenses for myopia control offer similar levels of axial length control. ${ }^{243}$

Arecent report of American Academy of Ophthalmology concluded that ortho-K may be effective in slowing myopic progression for children and adolescents, however, safety remains a concern because of the risk of potentially blinding microbial keratitis from contact lens wear. ${ }^{347}$

Customizing ortho-K lens designs to limit the central treatment zone may help to bring more plus power inside the pupil and achieve a greater shift in relative peripheral myopia. ${ }^{348-350}$ However, such approaches need to be evaluating in randomized controlled trials.

If customization of the ortho-K lenses is not possible, soft multifocal lenses are preferable for any patient who has less than $2.00 \mathrm{D}$ of refractive error. Also, patients who have a photopic pupil size smaller than $4.5 \mathrm{~mm}$ will be better served by a soft multifocal lens with a design that is independent of pupil size. Using soft multifocal contact lenses, the highest plus power that does not generate blur at distance without overminusing the original cycloplegic refractive power, was recommended by a recent myopia control summary. ${ }^{334}$

For orthokeratology lens wear should be encouraged every night for a minimum of $8 \mathrm{~h}$ per night to maximize correction for best-unaided vision during waking hours. ${ }^{339}$ The treatment effect of multifocal soft contact lens (MFSCL) is likely to be positively correlated with wearing time. ${ }^{230}$ Full time use of MFSCLs is recommended during school hours and for schoolwork at home, providing greater myopia control efficacy. ${ }^{339}$ Preferably, regulatory approved myopia controlling new designs, bifocal, progressive additional lenses (PAL), or single-vision spectacles may be prescribed for when children are not wearing their contact lenses. ${ }^{339}$

Another possibility is to add spectacles to supplement contact lens wear when accommodation is deficient. ${ }^{334}$ 
Children, who are intolerant of contact lenses or showing high exophoria at near, could be prescribed (prismatic) bifocal or anti-myopia spectacles. Fast progressors may not be treated sufficiently with low-add-power lenses, particularly in case of accommodative dysfunction.

Spectacle lenses are also the first option of care in very young children (who are unable to wear contact lenses due to access or cost), any situations that associated with poor hygiene conditions, or if children grew up in locations with no or only limited access specialized eye care..$^{351}$

Atropine therapy. A report from American Academy of Ophthalmology concluded that the use of atropine to prevent myopic progression is supported by Level I evidence. ${ }^{352}$ The World Society of Pediatric Ophthalmology and Strabismus in its Myopia Consesnus Statement argued that atropine $0.01 \%$ appears to offer an appropriate risk-benefit ratio, with no clinically significant visual side effects balanced against a reasonable and clinically significant $50 \%$ reduction in myopia progression (https://www.wspos.org/ wspos-myopia-consensus-statement/accessed 24 November 2020).

In a recent protocol developed by Chia and Tay, 353 children are first started on a lower dose of atropine with a plan to increase the dose as necessary. Once medication is started, progression (in terms of refraction and axial length) should be monitored every 6 months, for at least 2 years. Based on the protocol from the Netherland, ${ }^{93,337}$ axial length and gender-specific growth curve charts are used to evaluate the risk of myopipa/high myopia. Children with risk of myopia at the 75th percentile or above are then started on atropine $0.5 \%$ eye drops. ${ }^{337}$

The ATOM 2 study showed that $0.01 \%$ atropine resulted in a $60 \%$ risk of a refractive error rebound effect in children aged 8-10 years, compared to $30 \%$ at age $10-12$ years and $8 \%$ after the age of 12 years. ${ }^{281}$ The change in spherical equivalent was greater than the change in axial length and not directly associated with the change in axial length alone. ${ }^{281}$ This suggests that in children younger than 12 years who showed no progression in the past year, atropine $0.01 \%$ may be slowly tapered by reducing drop frequency (by 1-2 days/week each year). However, if children are older than 12 years, then the frequency of eye drops could be tapered more quickly (by 1-2 days/week every 6 months). Using this regime, most children will be off medication by about 1415 years of age..$^{353}$

The Low-Concentration Atropine for Myopia Progression (LAMP) study recommends the use of $0.05 \%$ atropine rather than $0.01 \%$, as the lower concentration allowed unacceptable levels of axial length progression. ${ }^{279}$ In children who progress on low-dose atropine, the frequency of application, or dose could be increased (using atropine $0.01 \%$ twice a day; or using a higher concentration, $0.05 \%, 0.1 \%, 0.5 \%$, or $1 \%)$. Increasing the dose of atropine needs to be balanced against side effects of loss of accommodation and glare/ aberrations from large pupil. Once an adequate control of myopia is achieved, medication can be continued till the child reaches teenage years and then tapered as required. There are some children $(11 \%)$, however, who may progress rapidly even on $0.5 \%$ atropine. ${ }^{331}$ If this occurs, then the possibility of stopping treatment or trying other treatment modalities should be discussed. Even after stopping treatment, it may be necessary to monitor children for a further 6-12 months to ensure that there is no further rebound. ${ }^{353}$

Patients undergoing atropine therapy will require distance refractive error correction. It is recommended that patients be prescribed their full distance refractive correction; however, patients may require near addition correction to alleviate near visual symptoms and photochromic lenses or additional sunglasses to relieve glare issues if necessary. ${ }^{339}$

Combination therapy in practice. For patients using mono-therapy in the form of atropine or ortho-K and who still experience progression of myopia and axial elongation at a faster rate than expected, combination therapy should be considered. Ortho-K with low-dose atropine improved myopia control by the synergistic effect compared with orthokeratology treatment alone, presumably because it increases the pupil area and, therefore, allows more plus power to reach the peripheral retina. ${ }^{328,330,334}$ The effectiveness and side effects of combination therapy with atropine and soft myopia control contact lens is unknown, and should be first evaluated in prospective clinical trials before being used in clinical practice. 


\section{TREATMENT DURATION}

Axial length is the most important metric to monitor in pre-myopic and myopic children. . $3,285,334,337,338^{2}$

Myopia generally progresses most rapidly during pre-teenager years (7-12 years), subsequently slowing through adolescence and adulthood. ${ }^{67,91,354}$ The mean age of myopia stabilization is around 15.6 years of age, and 95\% of myopes stabilize by age of 24 years. ${ }^{83}$

There are some publications of myopia onset and progression at a later age among specific occupational groups, during university education courses such as medicine, law or engineering. ${ }^{35-357}$

The efficacy of some treatments may wane after the first 6 months to 2 years of treatment. ${ }^{248,285,358-360}$ There is insufficient evidence that faster progressors, or younger myopes, derive greater benefit from treatment. ${ }^{285}$ The same treatments and protocols as applied in childhood may be applicable in later-onset myopia, although the available evidence is limited. ${ }^{339}$

In case of atropine treatment parents and patients should be made aware that myopia progression may accelerate after stopping higherdose atropine usage, but despite this rebound effect, the level of myopia post-treatment will be less than it would have been without treatment. ${ }^{280,282}$ The long-term use of atropine should only be undertaken with caution as long-term side effects have not been evaluated. ${ }^{339}$ It may be beneficial to tail off dosage or dose frequency at the end of treatment to minimize rebound effects. Although the results of the ATOM studies point to some loss of treatment efficacy with time, at least with the higher concentrations of atropine, a study by $\mathrm{Wu}$ and colleagues which involved concentrations between $0.05 \%$ and $0.1 \%$, suggested that treatment effects with low-dose atropine can be maintained for up to 4.5 years. ${ }^{287}$
Discontinuation of ortho-K lens wear before age 14 has been shown to lead to a more rapid increase in axial length over a 7-month period, faster than concurrent single vision spectacle wearing controls; however, this slows again with resumed lens wear after another 6 months. This suggests that ortho-K wear should not be discontinued before age $14 .{ }^{262}$

Long-term use of soft myopia control contact lens and ortho- $\mathrm{K}$ is not contraindicated if ocular health is maintained through regular aftercares and strong compliance. ${ }^{264,339,361}$ Progressive additional lenses can also be used for vision correction, but the long-term, clinically meaningful myopia control effect of such lenses is small in comparison with contact lens corrections, except in specific populations. ${ }^{145,214}$ Bifocal spectacle lenses might be a good solution for longevity treatment. ${ }^{362,363} \mathrm{~A}$ study of children wearing progressive addition lenses for 1 year, then switched to single vision glasses for 1 year showed no rebound. ${ }^{143}$ No rebound effect was reported with soft contact lens for myopia control. ${ }^{364}$

Compliance and safety issues may require a change in treatment modality or a halting of treatment. Poor tolerance of visual side effects may also prompt cessation or change of myopia control therapy. ${ }^{339}$

In conclusion, outdoor time is the most promising intervention method. There is consistent evidence of a benefit of slowing myopia development by the use of atropine eye drops, while the optimum concentration of atropine and the value of a combined use of atropine eye drops with optical devices are yet to be fully explored. There is also evidence of myopia control with soft multifocal contact lenses, orthokeratology, and new types of multifocal spectacle lenses. Information is constantly evolving, so it is important to stay abreast of studies published in the peer- reviewed literature.

Bibliogr. 364

Статията се публикува със съкращения със специалното разрешение на Европейското офталмологично общество 